\title{
Frequency Selective Surface Microwave Absorber for WLAN Applications
}

\author{
Thais C. Areias, Roberta N. G. Carvalho, Mauricio W. B. Silva, Leni, J. Matos, Member, IEEE, José Jaime G. \\ Peixoto Neto, Antonio Luiz P. S. Campos, Alfredo Gomes Neto
}

\begin{abstract}
Many researchers are studying electromagnetic wave absorbers. This is mainly due to the large number of existing wireless systems. The absorbers find numerous applications, from commercial to military systems. Allied to this, also the interest in Frequency Selective Surfaces (FSS), which are, basically, spatial filters, grows. Thus, this work proposes the use of FSS to design electromagnetic absorbers. In this study, Altair FEKO software was used. A parametric analysis is presented, demonstrating the understanding of the physical dimensions' effects. A prototype is built to validate the analysis performed. A good agreement between the numerical and experimental results is observed. Furthermore, measured results show that the absorber panel suppresses reflection (below - $10 \mathrm{~dB}$ ) from 1.97 GHz to $3.15 \mathrm{GHz}$, covering the entire ISM band.
\end{abstract}

Index Terms-Frequency selective surfaces, microwave absorber, WLAN

\section{INTRODUCTION}

$\mathbf{T}$ he increase in the number of electronic systems results in a corresponding growth in electromagnetic interference. These problems include false images, increased radar disorder, and reduced performance due to coupling between systems [1].

Microwave absorbers can be effectively used to minimize these types of problems. In some applications, such as in the military area, there is always a need to reduce the radar crosssection factor (RCS). As an example, one can cite the use of microwave absorber devices that play a key role in Stealth airplane technology [2].

For wireless security in buildings, traditional microwave absorbers, as Salisbury screen, can be placed in walls to provide isolation and reduce interference in nearby [3]. However, the ground plane of Salisbury screen may give rise to heavy reflections from its surfaces resulting in additional multipath, delay spread, and signal degradation [4].

Recently, we have observed an increase in the interest in using Frequency Selective Surfaces (FSS) in construction of microwave absorbers, since this type of structure allows an intelligent shielding, which absorbs only the frequency range of interest, becoming transparent to the other frequencies [5][9]. Therefore, with this structure we do not have additional

T. C. Areias, R. N. G. Carvalho, M. W. B. Silva, and L. J. Matos are with the Fluminense Federal University, Niteroi, Rio de Janeiro, Brazil (e-mails: thaiscarvalho@id.uff.br, robertang.carvalho@gmail.com, mauriciobenjo@id.uff.br, lenijm@id.uff.br).

J. J. G. P. Neto and A. L. P. S. Campos are is with Federal University of Rio Grande do Norte, Natal, Brazil (e-mails: josejaimegpneto@gmail.com, alpscampos@gmail.com).

A. G. Neto is with the Federal Institute of Paraíba, João Pessoa, Paraíba, Brazil (e-mail: alfredogomesjpa@gmail.com).

Digital Object Identifier: 10.14209/jcis.2020.23 multipath, delay spread, and signal degradation for the mobile communication frequencies, for example.

In this paper we propose a dual-layer FSS that is similar to a Salisbury screen with an important difference: the ground plane is substituted by a traditional FSS, which reflects only frequency range of interest. The advantage of the proposed structure is the use of a resistive layer, which does not spread, but absorbs signals in the frequency of interest, avoiding the increase of multipath, for example. A similar study was proposed by Rafique et al in [10], where unit cell circular patches were used to absorb WLAN signals in the $5 \mathrm{GHz}$ band. In contrast to the study proposed in [10], our work presents experimental evidence and makes use of an extensive parametric study to define the optimal dimensions of the structure.

\section{StATE OF ART}

Over the last few years, electromagnetic absorbers have attracted a massive attention from industry and academy for many applications, such as EMI/EMC issues, stealth techniques, among others [11]- [16]. Although the absorbers are well-defined engineering structures, many advances related to these structures are constantly published, with significant performance improvements presented. The main studies aim mainly to reduce volume and weight and maintain broadband absorption. In [15] the authors designed an ultra-wideband electromagnetic absorber based on the concept of metasurface Salisbury screen. The proposed absorber presented absorptivity above $88 \%$ from 3.74 to $18.5 \mathrm{GHz}$, which represents a fractional bandwidth of approximately $133 \%$. In addition, the structure exhibits angular stability and polarization insensitivity. To obtain performance improvement, the metal ground was replaced by a metasurface and genetic algorithm was used to optimize the elements of the metasurfaces.

An absorber based on the metamaterial concept for sensing applications of grain with a non-destructive approach in the microwave range was presented by [16]. To demonstrate the viability of the method, the authors performed simulations and measurements and showed that the structure can perform quality control of grain by measuring variations in the resonance frequency.

In [17] the authors used a hybrid electromagnetic absorber for the in-band radar cross section reduction of a Vivaldi antenna. Results showed reduction of monostatic RCS values up to $27 \mathrm{~dB}$ for both polarizations without significant change in the antenna radiation characteristics. 
The design of a metamaterial absorber, which can be used to improve the performance and reliability of UHF RFID systems, was proposed by [18]. The structure exhibits absorption over $90 \%$ across the band, with relative bandwidth of approximately $14 \%$, covering the entire band of the UHF RFID system. In addition, the structure is miniaturized and exhibits stability for TE e TM polarizations at low angles of incidence.

In [19], it was proposed the use of an absorber to improve electromagnetic environment pollution in a novel wireless inter/intra-chip communication channel system. The authors have shown that in addition to eliminating environmental pollution from the electromagnetic wave, the absorber layer can improve the propagation of the signal.

Another class of structures used to block electromagnetic signals is band-stop filters based on frequency selective surfaces. Studies have shown the shielding efficiency of these structures when applied in constructions, providing a radio secure environment. These structures allow the signal propagation in other bands, but may cause additional multipath or signal degradation due to reflection [20]- [22].

\section{Microwave AbSORbER DESIGN}

The structure of the electromagnetic absorber is illustrated in Fig. 1. The layers are printed on FR4 dielectric substrates with $180 \times 180 \mathrm{~mm}^{2}$ of area and relative electrical permittivity of 4.4. Bandstop filter characteristics are achieved by using a FSS of conducting square loops on a FR4 substrate with a thickness of $1.6 \mathrm{~mm}$. Physical dimensions of the square loops and the periodic spacing, are depicted in Fig. 1. The function of this conventional FSS layer is to act as a reflector for WLAN signals, at $2.45 \mathrm{GHz}$, while passing another service

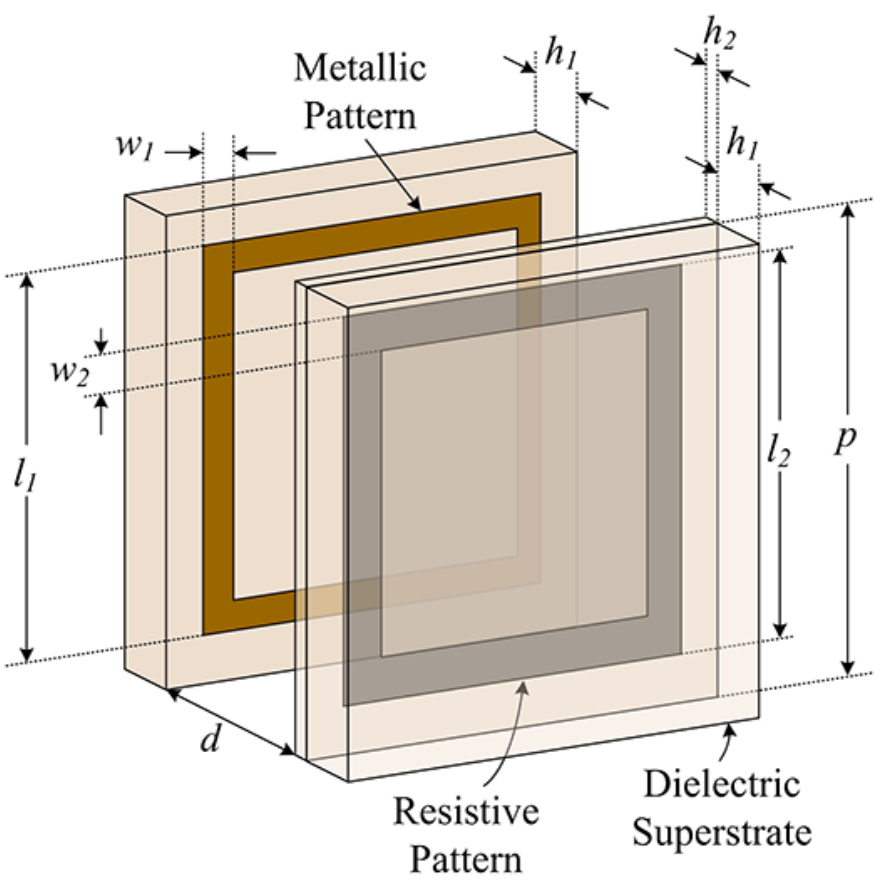

Fig. 1. Microwave absorber configuration composite by metal and resistive layers. signals. Then, the absorption characteristics are achieved by placing a second FSS layer consisting of resistive square loops, approximately $27 \mathrm{~mm}$ in front of the conducting FSS layer. A Nelco-4000 substrate, with a thickness of $0.254 \mathrm{~mm}$, dielectric constant of 3.6 and loss tangent of 0.009 , supported by a FR4 substrate was used and the surface resistance of resistive square loops is $R_{S}=50 \Omega / \square$.

The FSS microwave absorber is designed to absorb signals in the 2 to $3 \mathrm{GHz}$ frequency range. The resistive FSS absorbs the incident signals as well as the reflected signals from the conductive FSS. Other frequencies pass through the structure with a minimal or none interference. The resonance characteristic of the FSS depends mainly on the geometry. The square loop design was selected because it provides an angular stability and independence of polarization. In addition, the dielectric superstrate reinforces the angular stability characteristic for both, vertical and horizontal polarization without compromising absorption performance [23], [24]. A broad parametric study was carried out to evaluate how the design variables influence the frequency response of the microwave absorber, both from the point of view of reflection and transmission. The values of the parameters presented in this work were obtained based on this parametric study, performed in the software FEKO - Altair HyperWorks.

\section{PARAmETRIC ANALYSiS}

The resonance characteristic of the FSS depends mainly on the geometry. The square loop design was selected because it provides an angular stability and independence of polarization. In addition, the dielectric substrate reinforces the angular stability characteristic for both, vertical and horizontal polarization without compromising absorption performance [23], [24].

A parametric analysis was carried out to understand how the physical dimensions influence the frequency response of the transmission and reflection coefficients of the structure.

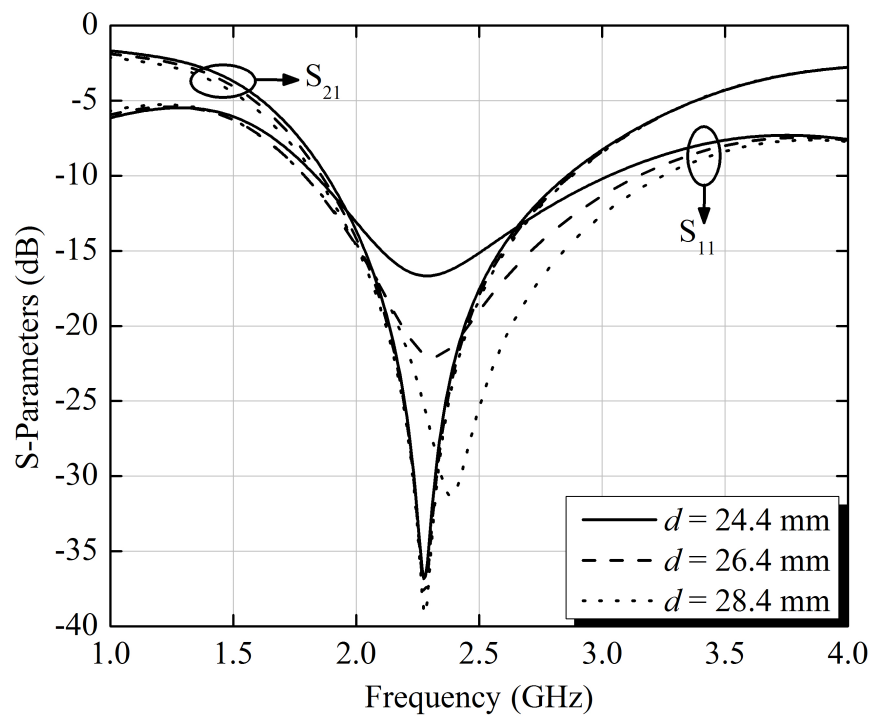

Fig. 2. S-parameters for different values of $d$. 


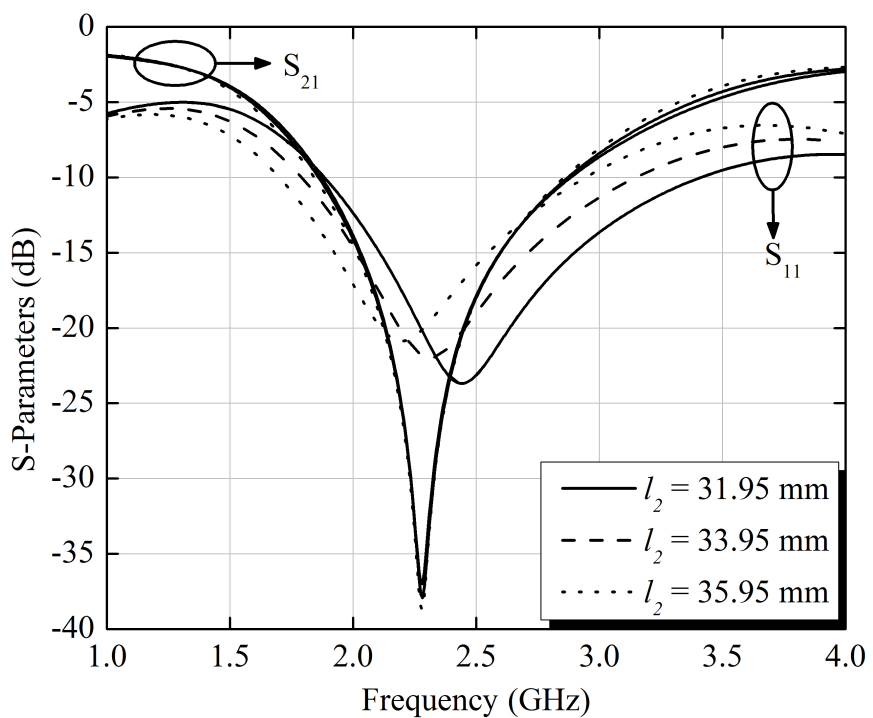

Fig. 3. S-parameters for different values of $l_{2}$.

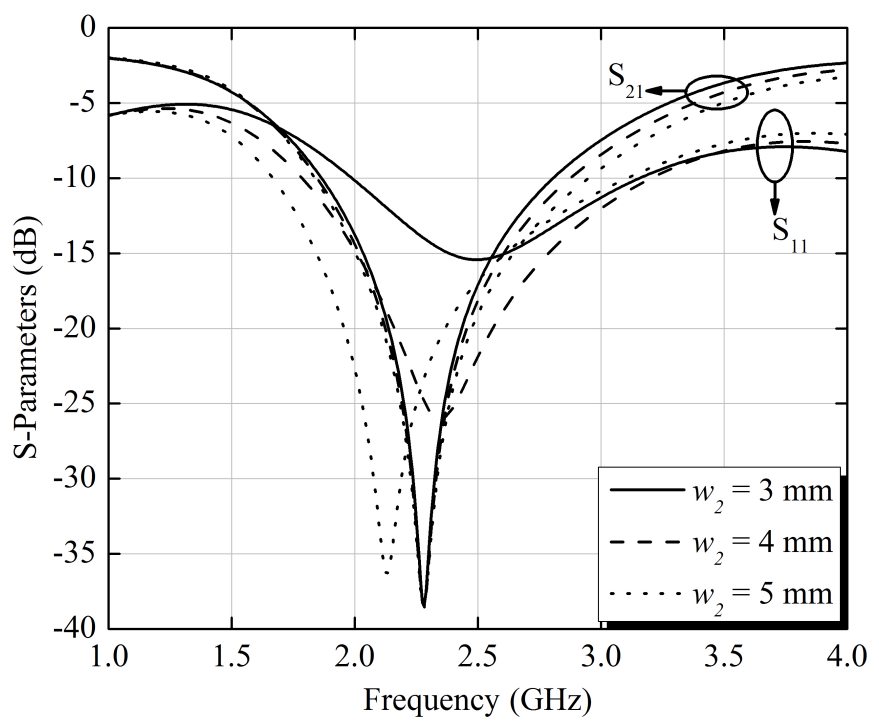

Fig. 4. S-parameters for different values of $w_{2}$.

An important parameter in design of this type of absorber is the distance of separation, $d$. Based on wavelength we chose three values for $d: 24.4 \mathrm{~mm}, 26.4 \mathrm{~mm}$, and $28.4 \mathrm{~mm}$. For all parametric analysis, the periodicity was $p=42.5 \mathrm{~mm}$ for both FSS and for all parametric analysis of resistive FSS, conducting layer has dimensions: $l_{1}=31.95 \mathrm{~mm}, w_{1}=2 \mathrm{~mm}$, and $h_{1}=1.6 \mathrm{~mm}$. Resistive layer has dimensions: $l_{2}=33.95$ $\mathrm{mm}, w_{2}=4 \mathrm{~mm}$, and $h_{2}=0.254 \mathrm{~mm}$. A FR 4 superstrate of 1.6 $\mathrm{mm}$ thickness was used for layer 2 . We can observe that $d$ has a great influence on bandwidth and resonance frequency, for the case of reflection coefficient. When $d$ increases, the bandwidth and resonance frequency increase, while the transmission coefficient remains, as illustrated in Fig. 2.

Another investigation is on the effect of square loop length, $l_{2}$, of the resistive layer. We chose three values for $l_{2}: 31.95$ $\mathrm{mm}, 33.95 \mathrm{~mm}$, and $35.95 \mathrm{~mm}$. Dimensions of conducting

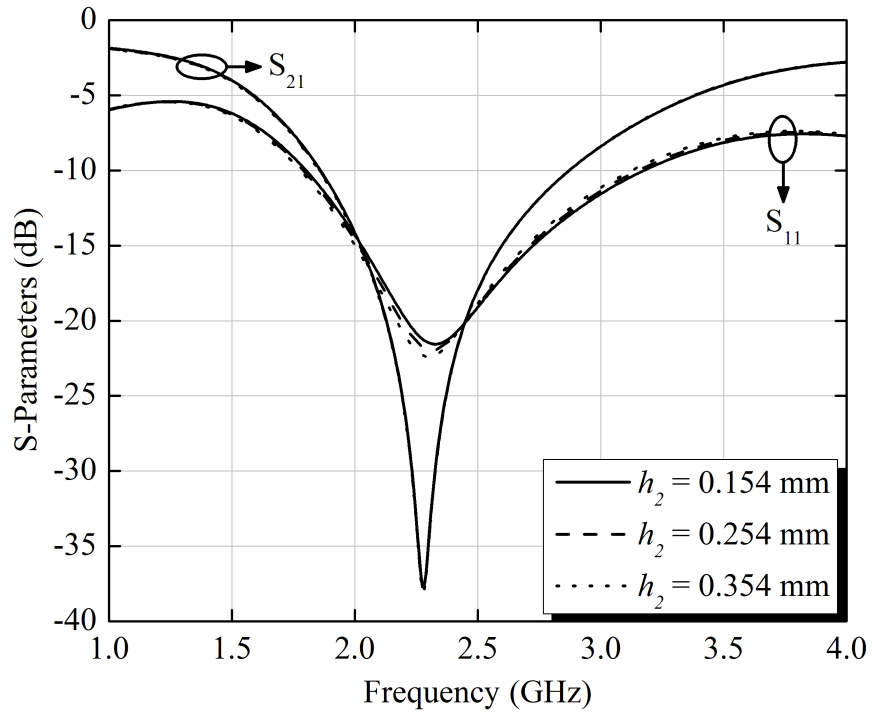

Fig. 5. S-parameters for different values of $l_{2}$.

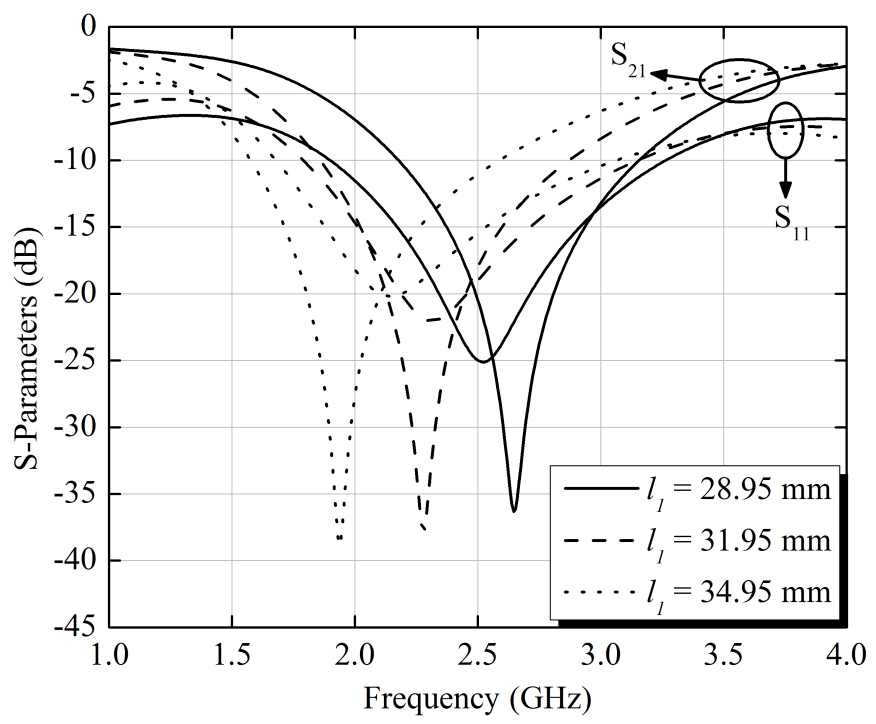

Fig. 6. S-parameters for different values of $l_{1}$.

layer remain. Dimensions of resistive layer are: $w_{2}=4 \mathrm{~mm}$, and $h_{2}=0.254 \mathrm{~mm}$. The gap between two layers was $d=$ $26.4 \mathrm{~mm}$. We can observe that $l_{2}$ has a medium influence on resonance frequency, while bandwidth almost did not change, for the case of reflection coefficient, while transmission coefficient remains, as illustrated in Fig. 3.

The third effect parameter analyzed was the width of the resistive square loop strip, $w_{2}$. We chose three values for $w_{2}$ : $3 \mathrm{~mm}, 4 \mathrm{~mm}$, and $5 \mathrm{~mm}$. Other dimensions of the resistive layer are: $l_{2}=33.95 \mathrm{~mm}$, and $h_{2}=0.254 \mathrm{~mm}$. The gap between two layers was the same of the past case. We can observe that $w_{2}$ has no influence about the transmission coefficient, while for reflection coefficient this parameter has a great influence on resonance frequency and bandwidth, when $w_{2}$ increases, resonance frequency and bandwidth decrease, as illustrated in Fig. 4. 


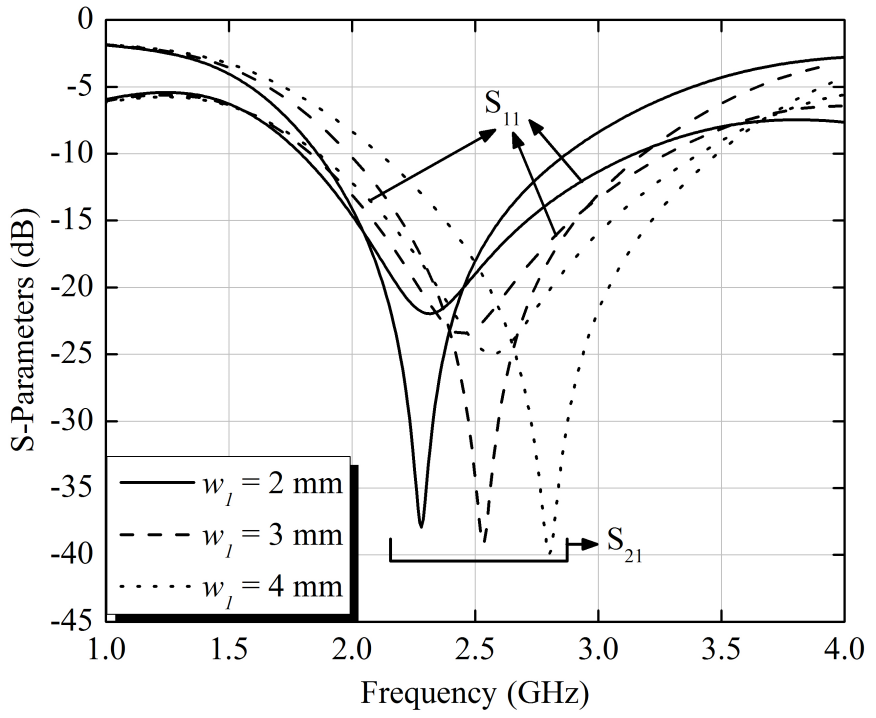

Fig. 7. S-parameters for different values of $w_{1}$.

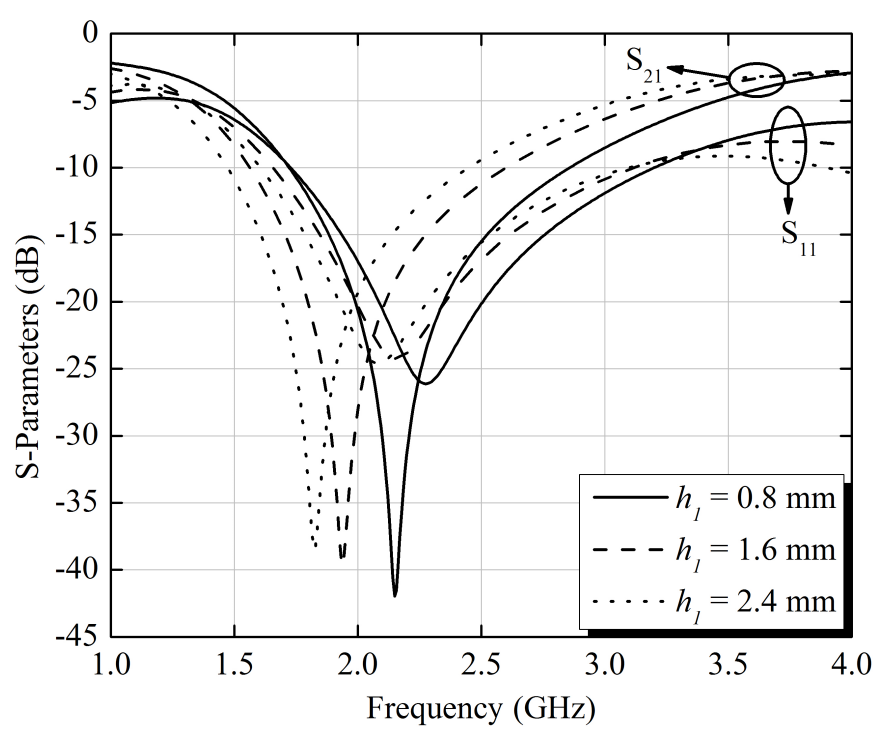

Fig. 8. S-parameters for different values of $h_{1}$.

The fourth effect parameter analyzed was the thickness of the dielectric resistive layer, $h_{2}$. We chose three values for $h_{2}: 0.154 \mathrm{~mm}, 0.254 \mathrm{~mm}$, and $0.354 \mathrm{~mm}$. For this parametric analysis, the resistive layer has dimensions: $p=42.5 \mathrm{~mm}, w_{2}=$ $4 \mathrm{~mm}$ e $l_{2}=33.95 \mathrm{~mm}$. The gap between layers was $26.4 \mathrm{~mm}$. The effect of $h_{2}$ on reflection and transmission coefficients is negligible, as illustrated in Fig. 5.

Now, we will do a parametric analysis of the conducting FSS. The effect of the conductive square loop length, $l_{1}$ is considered. We chose three values for this parameter: 28.95 $\mathrm{mm}, 31.95 \mathrm{~mm}$, and $34.95 \mathrm{~mm}$. For next analysis the resistive layer has dimensions: $p=42.5 \mathrm{~mm}, l_{2}=33.95 \mathrm{~mm}, w_{2}=4$ $\mathrm{mm}$ and $h_{2}=0.254 \mathrm{~mm}$. Conducting layer has dimensions: $w_{1}$ $=2 \mathrm{~mm}$, and $h_{1}=1.6 \mathrm{~mm}$. The gap between two layers was $26.4 \mathrm{~mm}$. As we can see, in Fig. 6, bandwidth and resonant frequency are strongly affected by $l_{1}$, for both reflection and

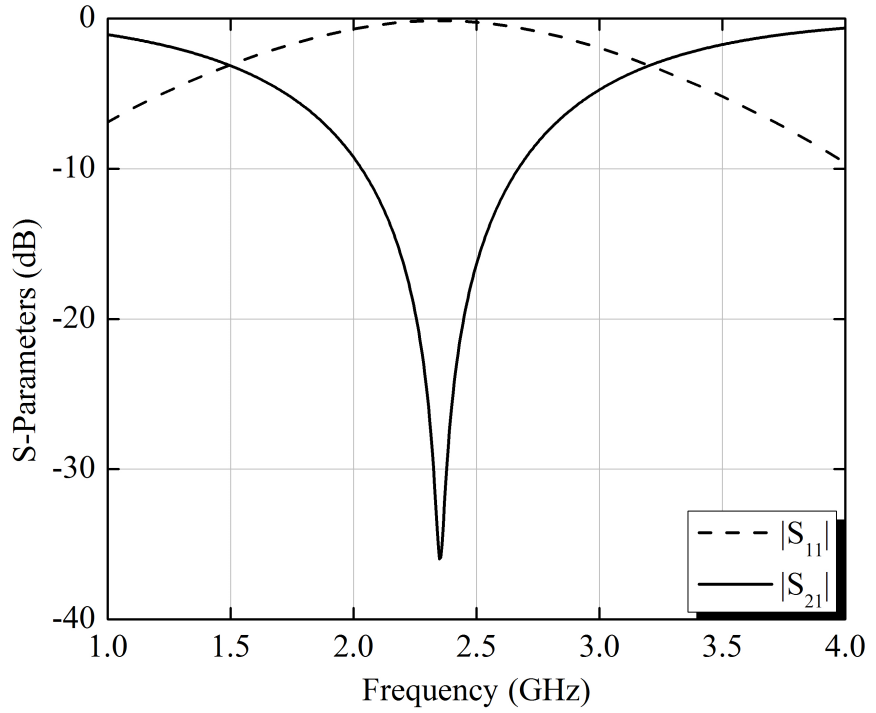

Fig. 9. S-parameters simulation of conducting FSS.

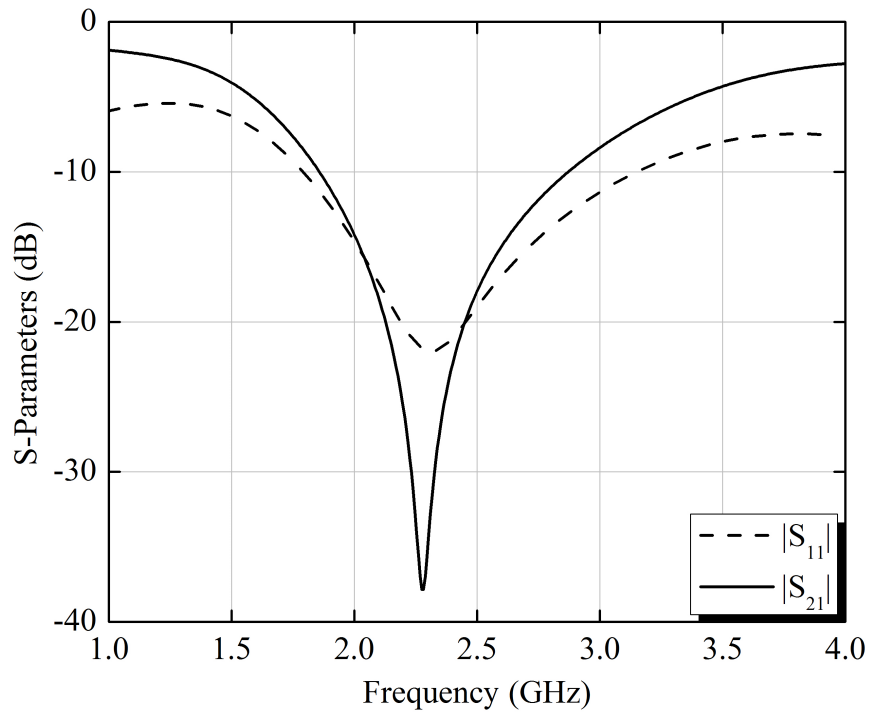

Fig. 10. S-parameters simulation of absorb/transmit structure.

transmission coefficients.

In Fig. 7 we can see the effect of conductive square loop strip width, $w_{1}$. We chose three values for $w_{1}: 2 \mathrm{~mm}, 3$ $\mathrm{mm}$, and $4 \mathrm{~mm}$. For this parametric analysis, conducting layer has dimensions: $l_{1}=31.95 \mathrm{~mm}$, and $h_{1}=1.6 \mathrm{~mm}$. The gap between two layers was $26.4 \mathrm{~mm}$. As we can see, bandwidth and resonance frequency are proportional to $w_{1}$, and a great variation in reflection and transmission coefficients is observed.

We also investigated the influence of the dielectric thickness of the conducting layer, $h_{1}$, on frequency characteristics. Three values were chosen for $h_{1}: 0.8 \mathrm{~mm}, 1.6 \mathrm{~mm}$ and $2.4 \mathrm{~mm}$. Other dimensions of conducting layer were: $l_{1}=31.95 \mathrm{~mm}$ and $w_{1}=$ $2 \mathrm{~mm}$. Resistive layer has dimensions: $p=42.5 \mathrm{~mm}, l_{2}=33.95$ $\mathrm{mm} w_{2}=4 \mathrm{~mm}$, and $h_{2}=0.254 \mathrm{~mm}$. The gap between two layers was $26.4 \mathrm{~mm}$. As we can see, in Fig. 8, the parameter $h_{1}$ has little influence on reflection coefficient and it has a 
moderate influence on transmission coefficient, reducing the resonance frequency and producing a negligible variation in bandwidth.

\section{Absorber Simulations and Measurements}

The structure dimensions were chosen to provide a more appropriate frequency response. Periodicity for both FSS, $p$, was $42.5 \mathrm{~mm}$. The dielectric used in the FSS conducting layer was the FR4, with dielectric constant $\varepsilon_{r}=4.4$ and thickness $h_{1}=1.6 \mathrm{~mm}$. For the resistive layer, a Nelco-4000 substrate with thickness $h_{2}=0.254 \mathrm{~mm}$ was used. The FSS layers are composed of square loop arrays. The conducting layer has dimensions: $l_{1}=31.95 \mathrm{~mm}$ and $w_{1}=2 \mathrm{~mm}$, while resistive layer is parameterized as: $l_{2}=33.95 \mathrm{~mm}$ and $w_{2}=4 \mathrm{~mm}$. In addition, between the two layers there is a separation $d=$ $26.4 \mathrm{~mm}$, which is in according to specification of a quarterwave distance for the frequency of operation used. The surface

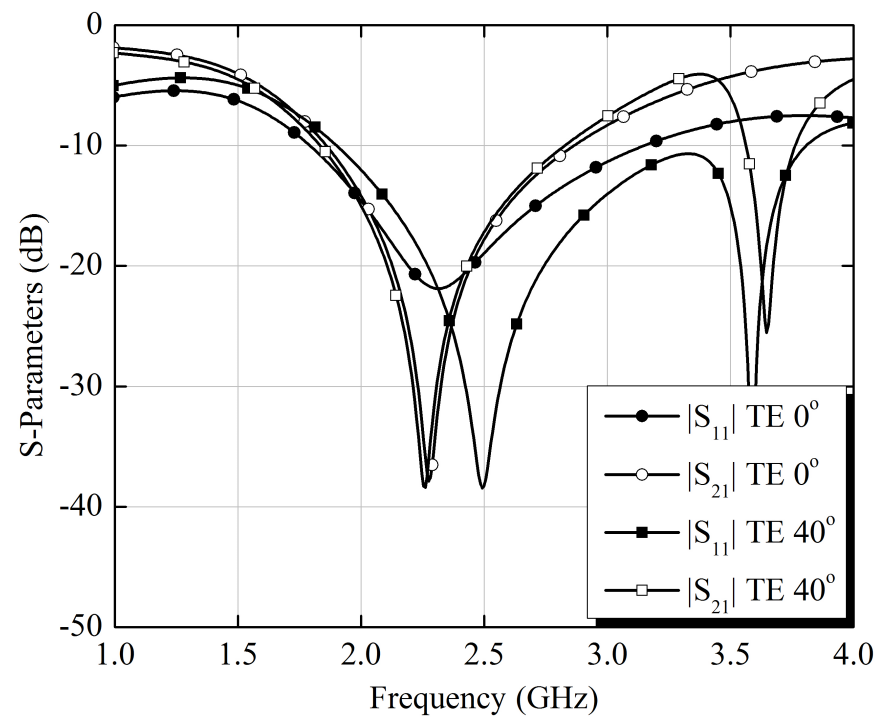

(a)

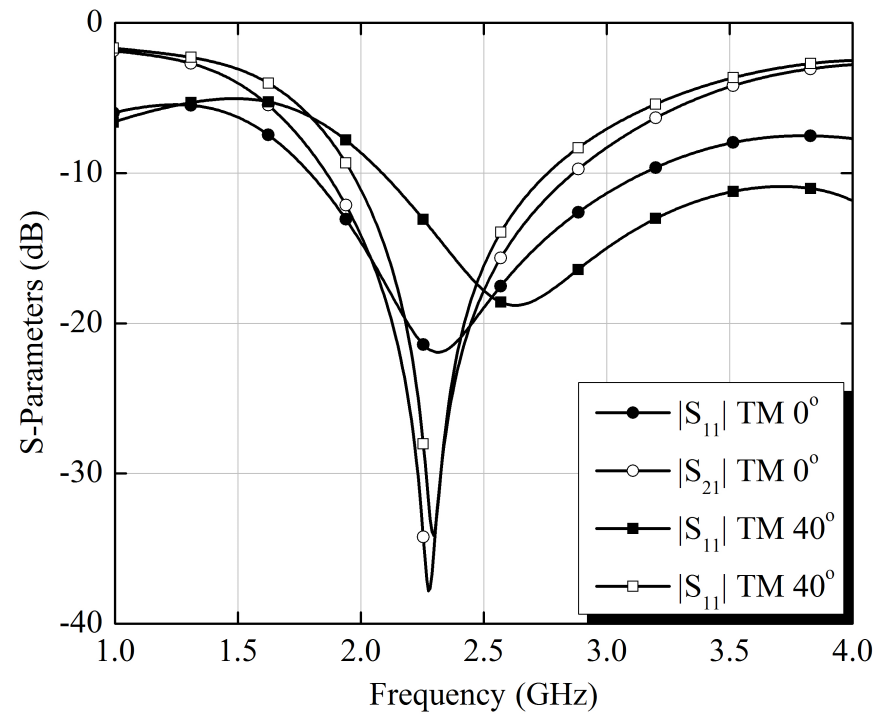

(b)

Fig. 11. S-Parameters under normal and oblique incidence for both (a) TE and (b) TM polarization. resistance of resistive layer is $R_{S}=50 \Omega / \square$. Finally, the resistive FSS has a FR4 dielectric superstrate of with thickness of $1.6 \mathrm{~mm}$ for support purposes.

To investigate the frequency characteristics of the conducting FSS structure, electromagnetic simulations were made, and they are show in Fig. 9. The results show the characteristic behaviour of a stop-band structure, with an attenuation pole as expected. As it can be seen, the FSS transmission coefficient magnitude is almost $-36 \mathrm{~dB}$ in a frequency close to $2.45 \mathrm{GHz}$, while the reflection coefficient magnitude remains around -0.2 $\mathrm{dB}$ which is a good insertion loss.

The result of the absorber FSS structure is shown in Fig. 10. It is clear from the results that the structure does not spread incoming signals, but attenuates waves through absorption. Simulated results show that the absorber panel suppresses reflection (below - $10 \mathrm{~dB}$ ) from $1.79 \mathrm{GHz}$ to $3.15 \mathrm{GHz}$. The resistive FSS design clearly provides absorption for the

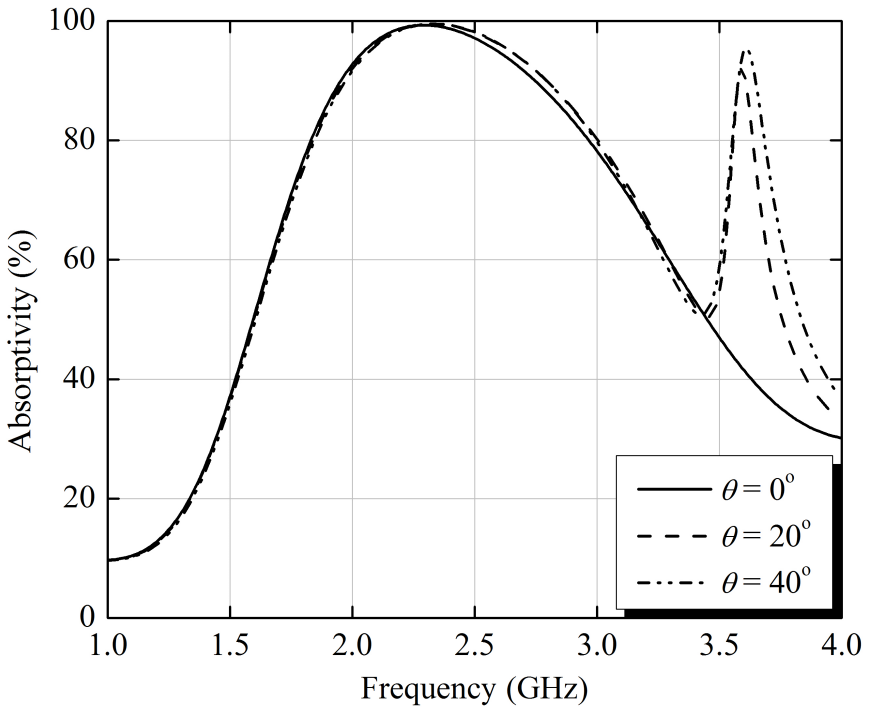

(a)

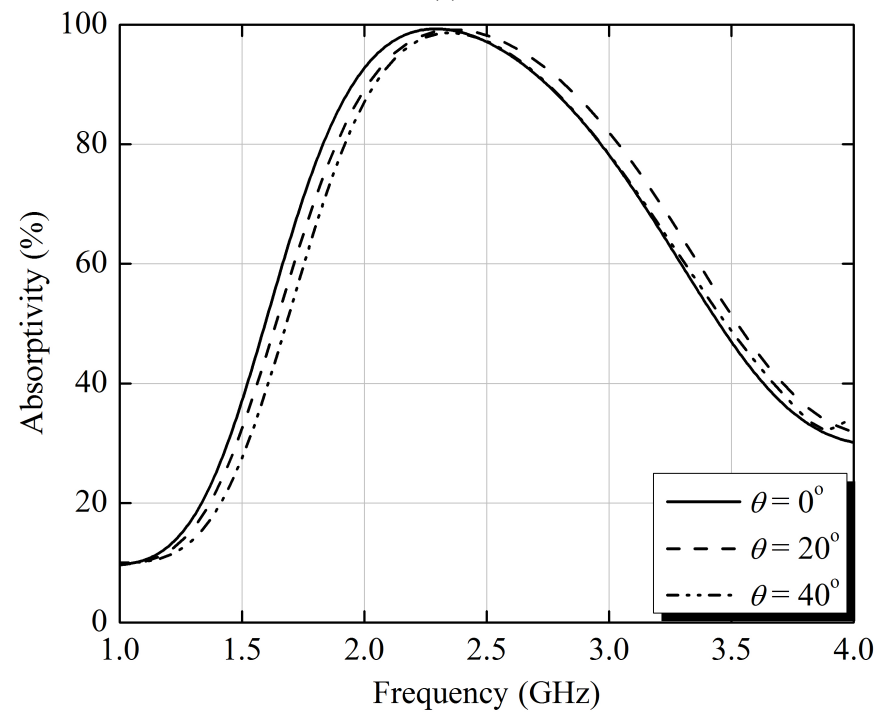

(b)

Fig. 12. Simulated absorptivity under normal and oblique incidence for both (a) TE and (b) TM polarization. 


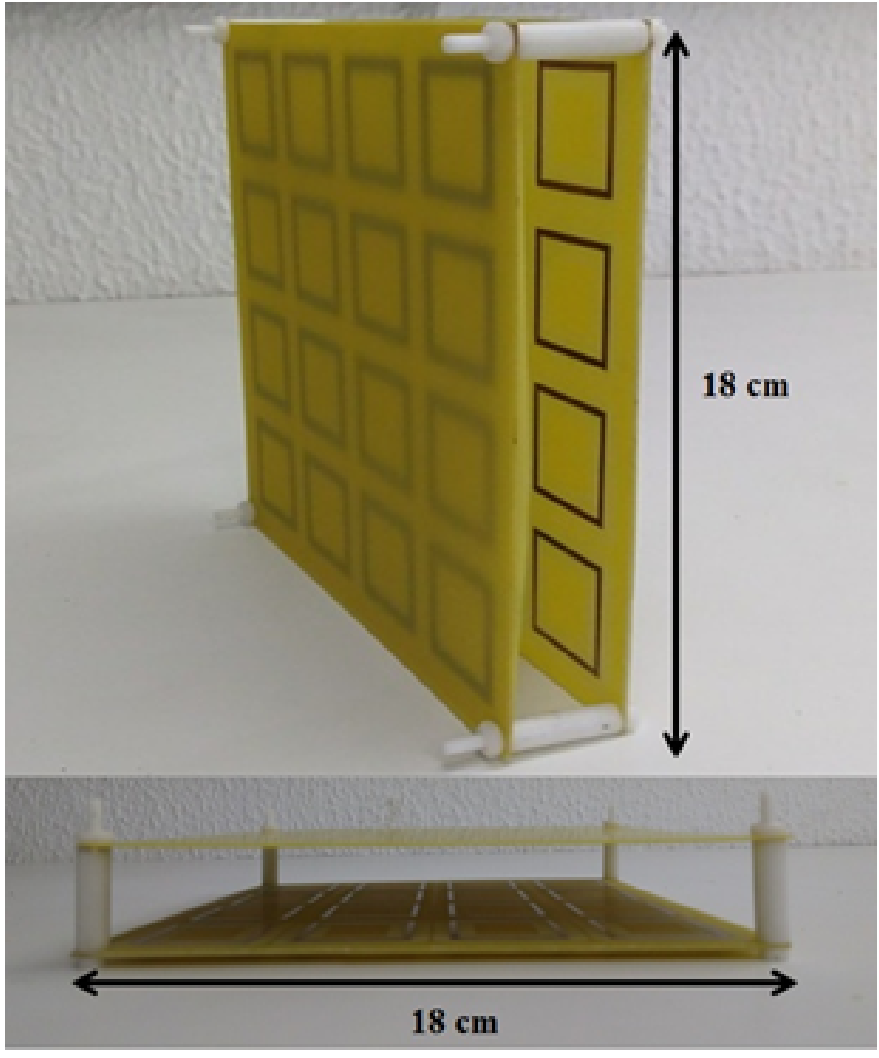

Fig. 13. Built absorber/transmit structure.

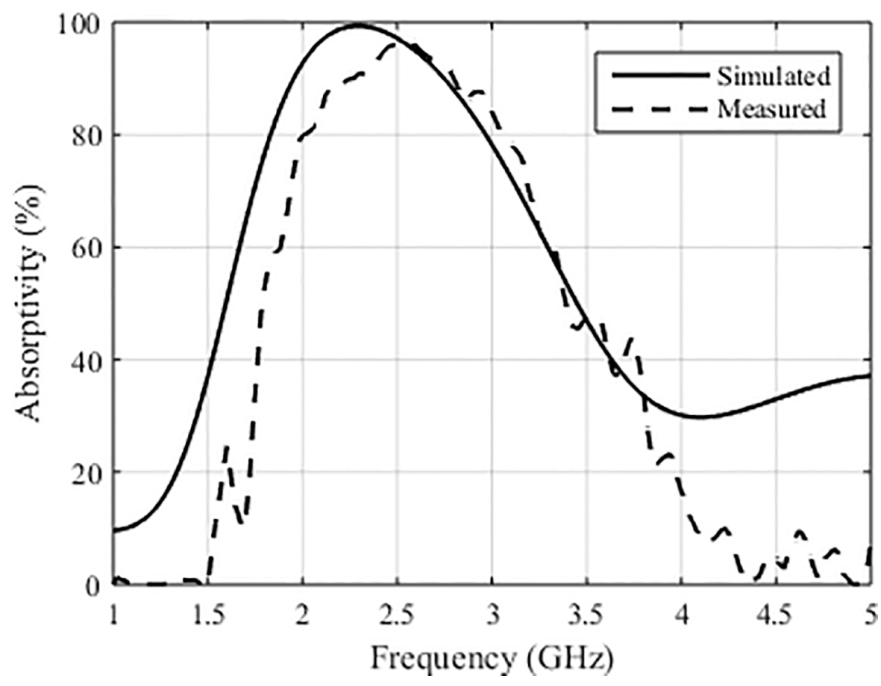

Fig. 14. Comparison between measured and simulated results for absorptivity.

reflected signals from the conducting FSS layer with maximum attenuation of $22 \mathrm{~dB}$ achieved at $2.28 \mathrm{GHz}$. The structure has a $-10 \mathrm{~dB}$ transmission bandwidth of $1.02 \mathrm{GHz}$. The absorber structure presents transmission coefficient magnitude of $-37.8 \mathrm{~dB}$ at $2.28 \mathrm{GHz}$. To verify the angle stability of the proposed structure, the response under oblique incidence was investigated. Fig. 11 shows the absorptivity for different incidence angles, for $\mathrm{TM}$ as well as TE polarization. As it can be observed in Figs. 11(a) and (b), the proposed absorber presents angular stability up to $40^{\circ}$ for the TE and

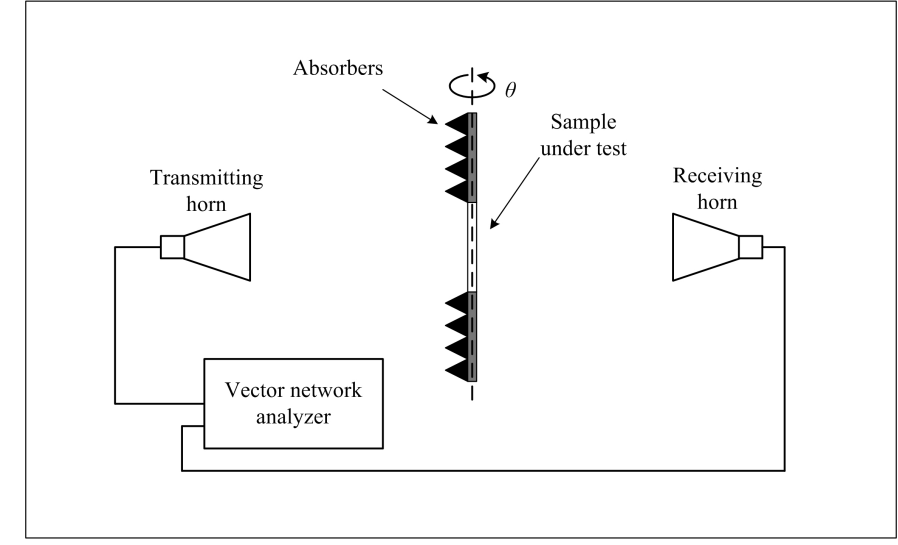

Fig. 15. Transmission coefficient measurement setup.

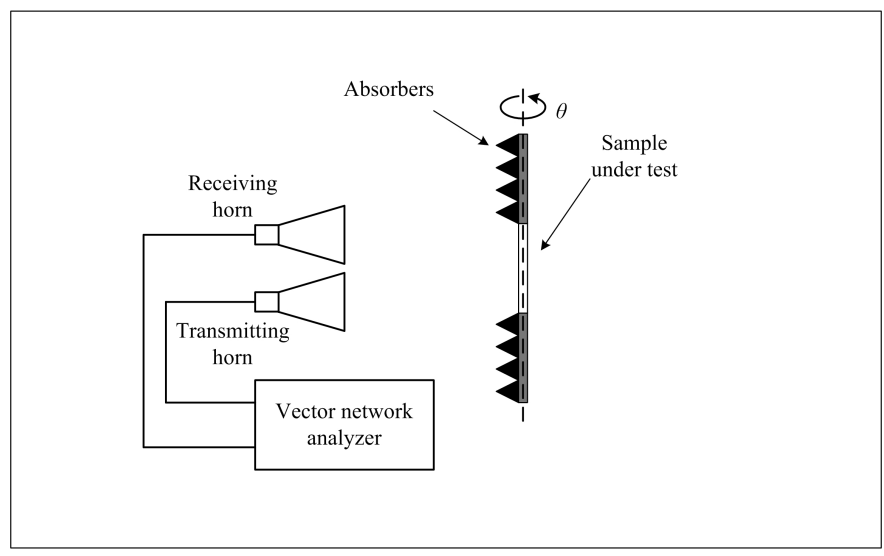

Fig. 16. Transmission coefficient measurement setup.

TM polarizations, respectively, with a frequency shift of the reflection coefficient in both polarizations.

To confirm the efficiency of the proposed absorber, we present the structure response in terms of the absorptivity. The total absorption can be quantified as follows [25]:

$$
A=1-R(\omega)-T(\omega)
$$

Where $R(\omega)=\left|R_{x x}\right|^{2}+\left|R_{x y}\right|^{2}$ is the reflected power and $T(\omega)=\left|T_{x x}\right|^{2}+\left|T_{x y}\right|^{2}$ is the transmitted power.

The co- and cross-polarized components are represented by " $x x$ " and "yx", respectively. In some absorbers the transmission can be ignored due metallic layer on the backside of the substrate. However, the proposed structure is composed of two frequency selective surfaces, therefore the co- and cross polarized components of the transmitted power cannot be ignored in the absorptivity calculation, using (1).

Fig. 12 shows the absorptivity for different incidence angles, $\theta$, for TM as well as TE polarization. The structure presents high absorption for normal incidence with peak at $2.28 \mathrm{GHz}$, corresponding to a maximum absorptivity of $99.66 \%$. Moreover, it is important to point out that the high absorptivity (>90\%) goes from 1.95 to $2.74 \mathrm{GHz}$, covering the entire ISM band. It is observed the structure maintains high absorption levels for oblique incidence angles up to $40^{\circ}$ for TE and TM polarizations, respectively, which shows wide-angle stability. 


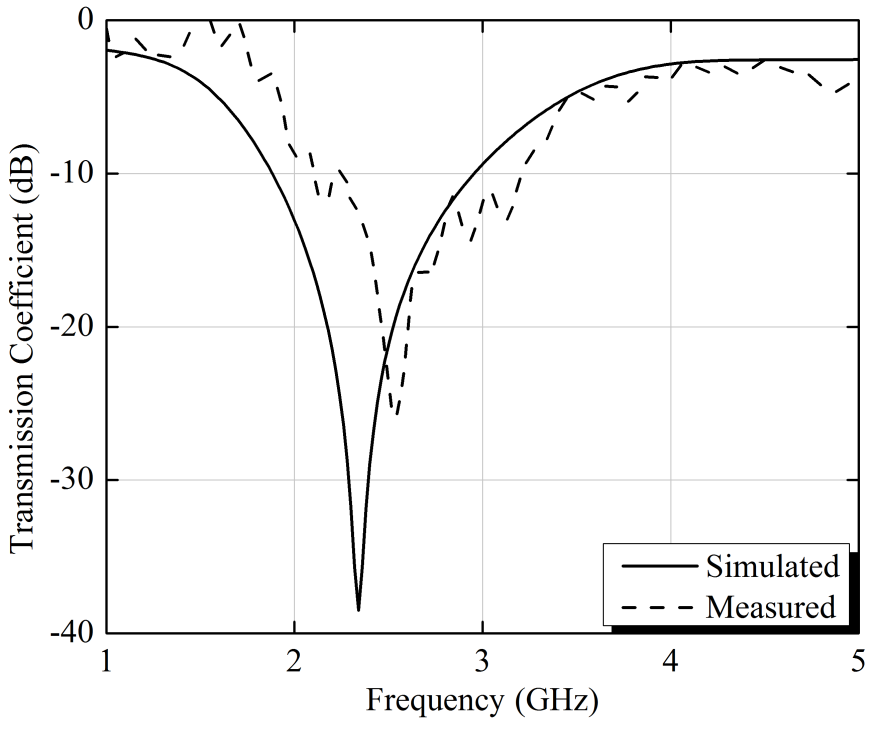

Fig. 17. Comparison between simulated and measured results for $S_{21}$ parameter.

The proposed unit cell is compact, once its periodicity and thickness are, in terms of the longest wavelength of the bandwidth of the absorption $\lambda_{L}, 0.27 \lambda_{L}$ and $0.19 \lambda_{L}$, respectively.

For validation purpose we built a conducting and a resistive FSS. The two FSS were cascaded using Teflon screws. Fig. 13 shows the built absorber.

Measured results were accomplished with the use of a vector network analyser from Agilent, model E5071C, and two horn antennas operating in the range of $0.7-18 \mathrm{GHz}$ with $16 \mathrm{dBi}$ of gain. To confirm the validity of the proposed structure, absorptivity measurements were performed and shown in figure 14, where it can be seen the agreement between measured and simulated curves. Reflection and transmission coefficients were measured. For transmission coefficient a traditional setup was used, as shown in Fig. 15. For reflection coefficient the setup shown in Fig. 16 was used. This kind of setup limits measurements of angular incidence.

In Fig. 17 we can see the comparison between simulated and measured results, for normal incidence and for the transmission coefficient. A good agreement between the results is observed. The simulated results show that the absorber panel suppresses reflection (below $-10 \mathrm{~dB}$ ) from $1.86 \mathrm{GHz}$ to $2.87 \mathrm{GHz}$. The measured results show that the absorber panel suppresses reflection (below - $10 \mathrm{~dB}$ ) from $1.97 \mathrm{GHz}$ to 3.15 $\mathrm{GHz}$. So, this absorption band is appropriated for the entire ISM band.

In Fig. 18 we can see the comparison between the simulated and measured results, for normal incidence and for the reflection coefficient. Again, a good agreement between the results is observed. Simulated results show that the absorber panel suppresses reflection (below - $10 \mathrm{~dB}$ ) from $1.79 \mathrm{GHz}$ to $3.15 \mathrm{GHz}$. Measured results show that the absorber panel suppresses reflection (below - $10 \mathrm{~dB}$ ) from $1.98 \mathrm{GHz}$ to 3.08 GHz. So, this absorption band is appropriated for the entire ISM band. Small differences between simulated and measured results are from the fabrication defects, because of an air

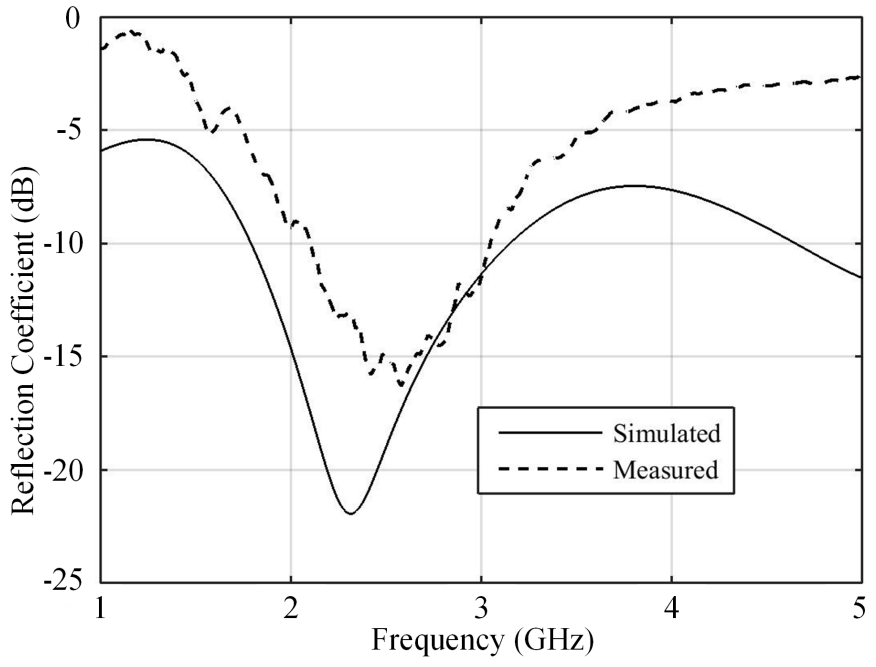

Fig. 18. Comparison between simulated and measured results for $S_{11}$ parameter.

gap between the very thin resistive layer and the dielectric superstrate.

\section{CONCLUSION}

The absorb/transmit frequency selective surface with a conductive FSS and resistive FSS, using square loops, presents a good performance, confirming the expected selective results in frequency of the ISM band and small dimensions. It is important to mention that although there was a high absorption in unwanted frequencies it does not generate any significant effect, because in this region there is no reflection, so the structure is totally transparent in other bands, such as Global System for Mobile Communication (GSM) and Universal Mobile Telecommunication System (3G). None multipath are produced in ISM band. The influence of each parameter on the response of the structure was shown through a wide parametric analysis. Moreover, simulated results demonstrated angle stability up to $40^{\circ}$ for the TE and TM polarization. The absorptivity of the absorber, under normal incidence, is the same for TE as well as TM polarization, which means that the structure is polarization insensitive. Measured results showed that the absorber suppresses reflection (below - 10 $\mathrm{dB}$ ) from $1.97 \mathrm{GHz}$ to $3.15 \mathrm{GHz}$. So, this absorption band is appropriated for the entire ISM band. Experimental results confirm the analysis developed.

\section{REFERENCES}

[1] R. N. Johnson, "Radar Absorbing Material: A Passive Role in an Active Scenario," in International Countermeasures Handbook, $2^{\text {nd }}$ ed., J. Peters, E.W. Communications, CA, 1992.

[2] F. Terracher and G. Berginc, "Thin electromagnetic absorber using frequency selective surfaces," IEEE Antennas and Propagation Society International Symposium. Transmitting Waves of Progress to the Next Millennium. 2000 Digest. Held in conjunction with: USNC/URSI National Radio Science Meeting (C, Salt Lake City, UT, 2000, pp. 846-849 vol.2, doi: 10.1109/APS.2000.875349.

[3] Y. Sha, K. A. Jose, C. P. Neo, and V. K. Varadan, "Experimental Investigations of Microwave Absorber with FSS Embedded in Carbon Fiber Composite," Microwave and Optical Technology Letters, vol. 32, no. 4, pp. 245-249, 2002, doi: 10.1002/mop.10144. 
[4] G. I. Kiani, A. R. Weily and K. P. Esselle, "A novel absorb/transmit FSS for secure indoor wireless networks with reduced multipath fading," in IEEE Microwave and Wireless Components Letters, vol. 16, no. 6, pp. 378-380, June 2006, doi: 10.1109/LMWC.2006.875589.

[5] G. I. Kiani, K. L. Ford, K. P. Esselle, A. R. Weily and C. J. Panagamuwa, "Oblique Incidence Performance of a Novel Frequency Selective Surface Absorber," in IEEE Transactions on Antennas and Propagation, vol. 55, no. 10, pp. 2931-2934, Oct. 2007, doi: 10.1109/TAP.2007.905980.

[6] Yilmaz, A. E. and M. Kuzuoglu, "Design of the square loop frequency selective surfaces with particle swarm optimization via the equivalent circuit model," Radioengineering, Vol. 18, no. 2, pp. 95-102, 2009.

[7] D. Ferreira, R. F. S. Caldeirinha, I. Cuiñas and T. R. Fernandes, "Square Loop and Slot Frequency Selective Surfaces Study for Equivalent Circuit Model Optimization," in IEEE Transactions on Antennas and Propagation, vol. 63, no. 9, pp. 3947-3955, Sept. 2015, doi: 10.1109/TAP.2015.2444420

[8] P. Munaga, S. Ghosh, S. Bhattacharyya, D. Chaurasiya and K. V. Srivastava, "An ultra-thin dual-band polarization-independent metamaterial absorber for EMI/EMC applications," 2015 9th European Conference on Antennas and Propagation (EuCAP), Lisbon, 2015, pp. 1-4.

[9] S. Piersanti et al., "Near-Field Shielding Performances of EMI Noise Suppression Absorbers," in IEEE Transactions on Electromagnetic Compatibility, vol. 59, no. 2, pp. 654-661, April 2017, doi: 10.1109/TEMC.2016.2626299.

[10] U. Rafique, G. I. Kiani, M. M. Ahmed and S. Habib, "Frequency selective surface absorber for WLAN security," Proceedings of the 5th European Conference on Antennas and Propagation (EUCAP), Rome, 2011, pp. 872-875.

[11] A. K. Singh, M. P. Abegaonkar and S. K. Koul, "Penta Band Polarization Insensitive Metamaterial Absorber for EMI/EMC Reduction and Defense Applications," 2017 IEEE MTT-S International Microwave and RF Conference (IMaRC), Ahmedabad, 2017, pp. 1-5, doi: 10.1109/IMaRC.2017.8449725.

[12] M. Agarwal and M. K. Meshram, "Isolation improvement of $5 \mathrm{GHz}$ WLAN antenna array using metamaterial absorber," 2016 URSI AsiaPacific Radio Science Conference (URSI AP-RASC), Seoul, 2016, pp. 1050-1053, doi: 10.1109/URSIAP-RASC.2016.7601144.

[13] V. K. Chakradhary, H. B. Baskey, R. Roshan, A. Pathik and M. J. Akhtar, "Design of Frequency Selective Surface-Based Hybrid Nanocomposite Absorber for Stealth Applications," in IEEE Transactions on Microwave Theory and Techniques, vol. 66, no. 11, pp. 4737-4744, Nov. 2018, doi: 10.1109/TMTT.2018.2864298.

[14] C. Martel, B. Gabard, F. Boust, V. Gobin and K. Elis, "A metamaterial electromagnetic wave absorber based on resistive films for micro-satellite spatial applications," 2018 IEEE International Symposium on Antennas and Propagation \& USNC/URSI National Radio Science Meeting, Boston, MA, 2018, pp. 441-442, doi: 10.1109/APUSNCURSINRSM.2018.8608739.

[15] Z. Zhou, K. Chen, B. Zhu, J. Zhao, Y. Feng and Y. Li, "Ultra-Wideband Microwave Absorption by Design and Optimization of Metasurface Salisbury Screen," in IEEE Access, vol. 6, pp. 26843-26853, 2018, doi: 10.1109/ACCESS.2018.2835815.

[16] Y. Zhang, J. Zhao, J. Cao, and B. Mao, "Microwave metamaterial absorber for non-destructive sensing applications of grain," Sensors, vol. 18, no. 6, p. 1912, Jun. 2018, doi: 10.3390/s18061912.

[17] H. B. Baskey, E. Johari and M. J. Akhtar, "Metamaterial Structure Integrated With a Dielectric Absorber for Wideband Reduction of Antennas Radar Cross Section," in IEEE Transactions on Electromagnetic Compatibility, vol. 59, no. 4, pp. 1060-1069, Aug. 2017, doi 10.1109/TEMC.2016.2639060.

[18] W. Zuo, Y. Yang, X. He, D. Zhan and Q. Zhang, "A Miniaturized Metamaterial Absorber for Ultrahigh-Frequency RFID System," in IEEE Antennas and Wireless Propagation Letters, vol. 16, pp. 329-332, 2017, doi: 10.1109/LAWP.2016.2574885.

[19] W. Wang et al., "Wireless Inter/Intra-Chip Communication Using an Innovative PCB Channel Bounded by a Metamaterial Absorber," in IEEE Antennas and Wireless Propagation Letters, vol. 15, pp. 1634-1637, 2016, doi: 10.1109/LAWP.2016.2519923.

[20] A. A. Dewani, S. G. O'Keefe, D. V. Thiel and A. Galehdar, "Window RF Shielding Film Using Printed FSS," in IEEE Transactions on Antennas and Propagation, vol. 66, no. 2, pp. 790-796, Feb. 2018, doi: 10.1109/TAP.2017.2780893.

[21] I. Lee, S. Cho, I. Hong and S. Yoon, "Improvement of communication performance in indoor environment using screen printed frequency selective film," 2017 IEEE Conference on Antenna Measurements \& Applications (CAMA), Tsukuba, 2017, pp. 120-123, doi: 10.1109/CAMA.2017.8273374.
[22] S. Cho, S. Lee, I. Lee and I. Hong, "Dual band frequency selective film design for indoor wireless LAN environment improvement," 12th European Conference on Antennas and Propagation (EuCAP 2018), London, 2018, pp. 1-4, doi: 10.1049/cp.2018.1167.

[23] P. Callaghan, E. A. Parker and R. J. Langley, "Influence of supporting dielectric layers on the transmission properties of frequency selective surfaces," in IEE Proceedings $\mathrm{H}$ - Microwaves, Antennas and Propagation, vol. 138, no. 5, pp. 448-454, Oct. 1991, doi: 10.1049/ip-h2.1991.0075.

[24] A.L.P.S. Campos, Superfícies seletivas em frequência: Anáalise e projeto, Editora do IFRN, 2010

[25] K. J. Vinoy and R. M. Jha, "Radar Absorbing Materials: from Theory to Design and Characterization," Kluwer Academic Publishers, Boston, 1996. doi:10.1007/978-1-4613-0473-9.

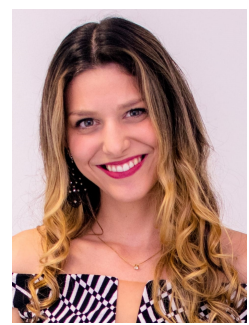

Thais Carvalho Areias received her bachelor degree in telecommunication engineering from Federal Fluminense University in 2018. Her field of interest includes Electromagnetism, Antennas, Microwaves, Frequency Selective Surfaces and Mobile Communication. She has experience in design and builds frequency selective surface and absorbers prototypes, on mobile network communication and analysis and development code from radio interface a layer and modem LSI and Qualcomm. Nowadays, she is working at SIDIA - Institute of Science and Technology as Protocol Software Developer.

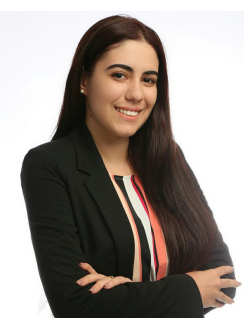

Roberta N. G. Carvalho is a Telecommunication Engineer. Her competence and engineering expertise lies in the field of applied electromagnetics, focusing on research and development of highfrequency printed circuit boards. Roberta has also participated in national scientific conferences related to RF/Microwave technologies, Optical Communications, and Signal Processing.

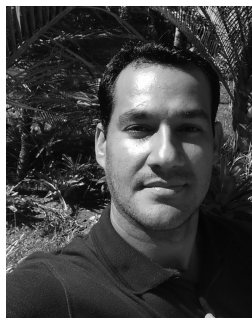

Mauricio W. B. Silva was born in Belem, Brazil, on October 25, 1980. He received the M.S. and $\mathrm{Ph} . \mathrm{D}$. degrees in electrical engineering from the University of Campinas, Campinas, Brazil, in 2009 and 2014, respectively. From 2015 to 2016, he was a Postdoctoral Researcher with the Department of Telecommunications Engineering at the Fluminense Federal University. He is currently a Professor with the Department of Telecommunications Engineering, Fluminense Federal University. His current research interests include metamaterials, applied electromagnetics, radio frequency identification systems, and antennas. 


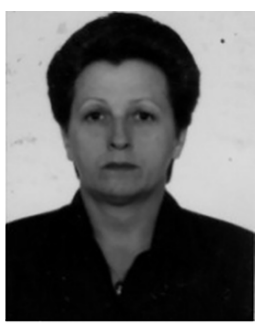

Leni J. Matos was born in Rio de Janeiro, RJ, Brazil on January 2, 1954. She earned her B.S. in Electronics and Telecommunication Engineering at Rio de Janeiro State University, Rio de Janeiro, Brazil, in 1977. In 1981 she earned her M.S. degree at the Military Institute of Engineering (IME; Brazil), and in 2005 she earned her D.Sc. degree at the Center of Telecommunication of Pontifical Catholic University of Rio de Janeiro (CETUC/PUC/RJ, Brazil). Since 1980, she has been teaching graduate and postgraduate courses at various universities. She is currently a professor and researcher at Fluminense Federal University (UFF), Brazil. Her research interests include mobile radio channel and underwater characterization, modeling and simulation; antennas and propagation. She is member of the Institute of Electrical and Electronics Engineers (IEEE).

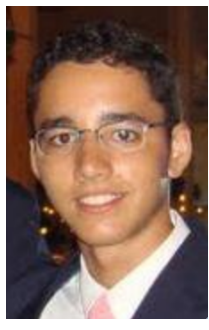

José Jaime G. Peixoto Neto received the B.Sc. degree in Science and Technology from the Federal University of Rio Grande do Norte (2012), B.Sc. degree in Telecommunications Engineering from the Federal University of Rio Grande do Norte (2014) and a master's degree in Electrical Engineering from the Federal University of Rio Grande do Norte (2016). Has experience in Electrical Engineering, focusing on Telecommunications, acting on the following subjects: absorbers, frequency selective surface and angular stability.

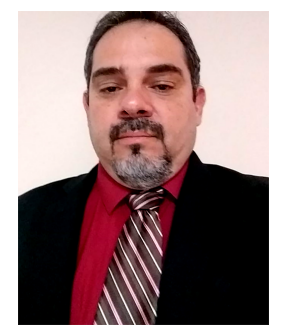

Antonio L. P. S. Campos received the B.S.E.E. and M.S.E.E. degrees from the Federal University of Rio Grande do Norte, Natal RN, Brazil, in 1996 and 1999, respectively, and he received the Ph.D. degree from Federal University of Paraíba, Campina Grande PB, Brazil. Since 2010, he has been with the Federal University of Rio Grande do Norte, Natal $\mathrm{RN}$, Brazil, where he is currently a Professor of the Communication Engineering Department. He is currently involved in the areas of FSS, scattering of electromagnetic waves, antennas, and planar devices. $\mathrm{He}$ is a member of the Brazilian Microwave and Optoelectronics Society (SBMO).

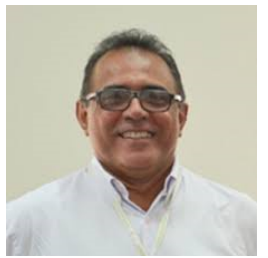

Alfredo Gomes Neto received the B.Sc. degree from Federal University of Paraíba, Campina Grande, Brazil, 1986, M.Sc., 1989, and D.Sc., 1994, all in electrical engineering and in microwave area, from the same University. In 1993, he held part of his D.Sc. at the ENSEEIHT, INPT, Toulouse, France, to where he returned in 2005 , during the post-doctorate researches. Since 1989 he is with the Federal Institute of Paraíba, IFPB. In 1994 he was a founder of the Group of Telecommunications and Applied Electromagnetism, GTEMA, at the IFPB. $\mathrm{He}$ is a member of the Brazilian Society of Microwaves and Optoelectronics, SBMO, and IEEE Senior Member. His research interests include electromagnetic theory, microwaves, wave propagation, FSS, antennas and numerical methods. 